\title{
The Role of Health Beliefs Model in Predicting Prostate Cancer Screening in a Group of Men Over Age Fifty in Shiraz
}

\author{
Abdulaziz Aflakseir ${ }^{1, *}$ \\ ${ }^{1}$ Department of Clinical Psychology, School of Education and Psychology, University of Shiraz, Shiraz, IR Iran \\ "Corresponding author: Abdulaziz Aflakseir, Department of Clinical Psychology, School of Education and Psychology, University of Shiraz, Shiraz, IR Iran. E-mail: \\ aaflakseir@shirazu.ac.ir
}

Received 2015 July 05; Accepted 2016 June 28.

\section{Dear Editor,}

Screening of prostate cancer has been shown to reduce mortality rate [1]. Health belief model has been related to participation in health behaviors such as prostate cancer screening. The Health belief model consists of the following components: (a) perceived seriousness; (b) perceived susceptibility; (c) perceived barriers; (d) perceived benefits; and (e) health motivation [2]. The aim of this study is to predict prostate cancer screening based on the components of health belief model among men over age 50 years. In this correlational study, 150 individuals with mean age $67.3 \pm 7$ years and ranges of 53 to 80 years were recruited from the public places in Shiraz using convenience sampling. The health belief model scale - prostate cancer screening was used to measure health beliefs regarding prostate screening consisting five components including susceptibility perception, seriousness perception, health motivation, barrier perception, and benefit perception. Data was analyzed by SPSS -16. Findings indicated that about 7.5\% of the sample performed prostate examination (DRE and PSA). According to the research data, about $20 \%$ of individuals who practiced prostate screening had a history of prostate cancer in family. Seventeen percent of the sample endorsed that they had enough knowledge about examination procedures, $64 \%$ of the sample had little knowledge about the examination, and $19 \%$ believed that they did not have knowledge on examination. In terms of the components of health belief model, results showed that $35 \%$ of sample felt that they were susceptible to prostate cancer, $83 \%$ perceived prostate cancer to be a serious disease, $19 \%$ believed that there was barriers to perform screening and $78 \%$ believed that prostate cancer screening was beneficial. Logistic regression analysis indicated that three of the independent variables predicted prostate cancer screening. Having prostate cancer in family had a significant contribution in predicting prostate cancer screening $(\beta=.35, \mathrm{P}<.01)$. Among dimensions of the HBM, two dimensions including perceived seriousness $(\beta=.21, \mathrm{P}<.01)$ and perceived benefits $(\beta=.19, \mathrm{P}<.01)$ had a significant contribution in prediction of screening performance. In other words, individuals who had prostate cancer in family; felt prostate cancer as a serious illness and perceived prostate cancer screening beneficial were more likely to participate for screening. The proportion of prostate cancer screening in this study was lower than developed countries and a little higher than some developing countries [3]. An explanation for low attendance for screening may be lack of access to health service. This study suggests that health system should provide educational interventions to improve the knowledge of men on prostate cancer screening.

\section{References}

1. Cookson MM. Prostate cancer: screening and early detection. Cancer Control. 2001;8(2):133-40. [PubMed: 11326167].

2. Alder B. Psychology of Health: Application of psychology for health professionals. Harwood academic; 1995.

3. Woolf SH, Krist AH, Johnson RE, Stenborg PS. Unwanted control: how patients in the primary care setting decide about screening for prostate cancer. Patient Educ Couns. 2005;56(1):116-24. doi: 10.1016/j.pec.2003.12.002. [PubMed: 15590232]. 